\title{
Inverse estimation of terminal connections in the cardiac conduction system
}

\author{
Fernando Barber ${ }^{a}{ }^{*}$, Miguel Lozano a , Ignacio García-Fernández a , \\ Rafael Sebastian ${ }^{a}$
}

Modeling the cardiac conduction system is a challenging problem in the context of computational cardiac electrophysiology. Its ventricular section, the Purkinje system, is responsible for triggering tissue electrical activation at discrete terminal locations, which subsequently spreads throughout the ventricles. In this paper we present an algorithm that is capable of estimating the location of the Purkinje system triggering points from a set of random measurements on tissue. We present the properties and the performance of the algorithm under controlled synthetic scenarios. Results show that the method is capable of locating most of the triggering points in scenarios with a fair ratio between terminals and measurements. When the ratio is low, the method can locate the terminals with major impact in the overall activation map. Mean absolute errors obtained indicate that solutions provided by the algorithm are useful to accurately simulate a complete patient ventricular activation map.

Copyright (C) 2016 John Wiley \& Sons, Ltd.

Keywords: Cardiac Conduction System; Purkinje Tree; Non-statistical estimation; Approximation

\section{Introduction}

Computational modelling of the heart aims at helping to understand the complex structure and function of the heart in health and disease. The construction of realistic computational cardiac models that can be personalized to a patient is challenging and requires the fusion of disparate medical and biological data [1, 2]. On the one hand, clinical images from computed tomography (CT) or magnetic resonance imaging (MRI) can be acquired and segmented to build patient-specific 3D heart geometries. Those segmented hearts would be meshed to create a fine computational domain in which finite element methods can be applied. On the other hand, to model heart function ex-vivo measurements from cell and tissue samples are needed to feed models, which are commonly represented by sets of differential equations that describe its coupled nonlinear behaviour. However, the heart is highly complex and inhomogeneous, and not all the cardiac structures and tissue properties can be personalized due to imaging resolution limits. Among these structures it is the cardiac conduction system (CCS) or Purkinje tree (in the ventricles), which is responsible for the synchronized activation of the cardiac muscle that triggers the heart contraction. In a healthy human heart, the CCS functions as a 'highway' placed in the inner cardiac surface, where the electrical signals travel fast up to the Purkinjemyocardial junctions (PMJs). At each PMJ the impulse enters the 'working' contractile myocardium, which slowly propagates the electrical signal as a wavefront activating the heart tissue. Once a given cell has been activated, it cannot be activated again

\footnotetext{
a Departament d'Informàtica, ETSE, Universitat de València

* Correspondence to: Fernando Barber, Departament d'Informàtica, ETSE, Universitat de València, 46100 Burjasot (Valencia), Spain. E-mail: fernando.barber@uv.es
} 
during a certain period (refractory period) by a second electrical wavefront. As a result, not all PMJs are effective, in the sense that some might try to trigger cardiac tissue that has been already activated. Therefore, they have no practical effect, or their contribution is masked by other surrounding PMJs.

The structure of the Purkinje tree and the location of the PMJs cannot be directly segmented from any in-vivo imaging technique. This fact has led researchers to the construction of generic population-based computational models of the CCS in the ventricles [3, 4]. Ex-vivo images has also been used to build reference models [5, 6]. The reader is referred to [4] for a review on different techniques commonly used to build computer models of the CCS. Recently, some techniques have been developed to infer the location of PMJs and structure of a Purkinje tree from electro-anatomical maps (EAMs) acquired in-vivo in the clinic $[7,8,9]$ during radio frequency ablation (RFA) interventions. In an EAM procedure, the local activation time (LAT) at different tissue locations can be measured using catheters on the inner surface of the ventricles. EAMs are one of the few in vivo clinical sources of information that can be directly used to obtain the electrical function of the heart.

In the works by [8] and [9], a mathematical method to optimize positions of randomly placed PMJs is presented that reduces simulated activation errors given a precomputed generic Purkinje tree structure. In [7], the location of PMJs is determined from singularity points on highly dense activation maps obtained from simulations. None of those techniques try to estimate the real location of PMJs, but a distribution of them that is coherent with tissue global activation sequences. Estimating the real location of heart electrical triggers, i.e. PMJs or ectopic foci in pathological tissue, is highly relevant for both constructing realistic models of the heart and providing substrate data to planning interventions. For instance the CCS is known to interact retrogradely with the electrical impulse triggered by pacing leads of cardiac resynchronization therapy devices [10, 11]. In addition, it is still unknown how ablation lines carried out in ventricular tissue to interrupt ventricular tachycardia can affect CCS and in particular PMJs, and therefore a better knowledge in their localization might help in planning interventions.

Our goal is to perform an inverse estimation of the location and activation time of PMJs on the inner surface of the heart, given a set of randomly distributed measurements, similar to those obtained in EAMs. In this work, we present an estimation method, and evaluate it on several synthetic scenarios. To represent the complex Purkinje network patterns described in the literature [12], we build synthetic tree structures recursively. Basically, we create two perpendicular child branches at the end of each branch with decreasing length and locating the PMJs at the leaf nodes of the tree. We build several scenarios by constructing Purkinje trees with an increasing number of branches and PMJ density, to assess the potential of the algorithm to estimate PMJs. The estimation of the underlying Purkinje system branching structure is out of the scope of this study.

\section{PMJ estimation method}

To test the methodology we take the following assumptions: the cardiac tissue is represented by a $2 \mathrm{D}$, euclidean domain, $\Omega \subset \mathbb{R}^{2}$; the signal propagation is considered isotropic, and the propagation velocity is constant. According to the previous description of a Purkinje tree, we assume that the signal enters cardiac tissue through a set $\mathcal{S}$ of $n$ PMJs, with locations $\mathbf{s}_{k} \in \Omega, k=1, \ldots, n$. The activation time of PMJ $k$ will be denoted as $\tau_{k} \in \mathbb{R}$.

Given a point $\mathbf{x} \in \Omega$, its local activation time (from now on LAT) will be the earliest arrival time of the signal from the source nodes, i.e. PMJs, and is given by

$$
t(\mathbf{x})=\min _{k}\left(\tau_{k}+\frac{\left\|\mathbf{x}-\mathbf{s}_{k}\right\|}{v}\right)
$$

where $v$ is the propagation velocity of the signal through the cardiac tissue. Figure 1 (a) shows a simple scenario with two PMJs represented with solid circles, and several points with measurements (LATs) represented with crosses. Three of the measurement points displayed with LATs $t_{i 1}, t_{i 2}$ and $t_{i 3}$, were activated by the same PMJ (indicated with arrows).

Since the Purkinje tree cannot be observed, PMJs will be considered as unknown, both in their number and location. Our goal is to estimate the location of the set of effective PMJs that produces the observed measurements, which are the activation times at $m$ given locations. Thus, we state our problem as 

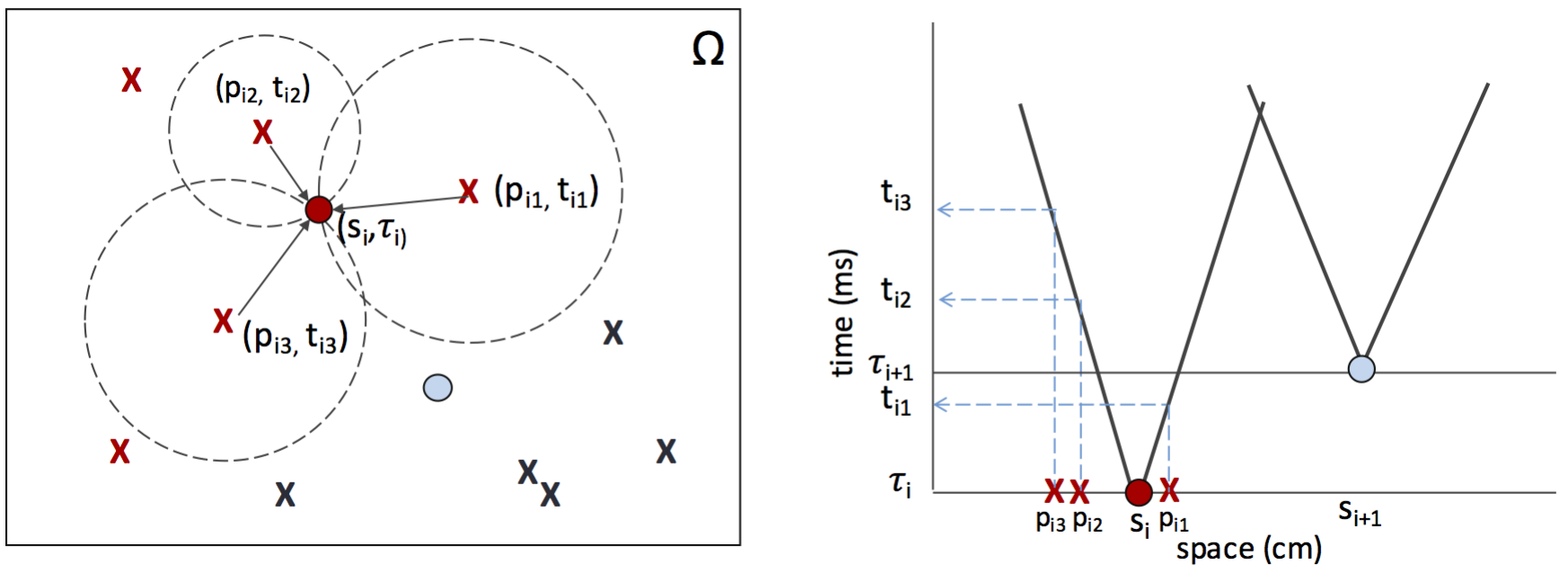

Figure 1. The relationship between PMJs and measurement points. Spatial representation in a 2D plane with spatial location of PMJs and measurement points (left) and a space-time representation (right). If a location is activated by a certain PMJ, $s_{i}$, then its space-time coordinates ( $p_{i}, t_{i}$ ) belong to a cone with vertex in the PMJ and with slope defined by the propagation velocity.

Problem 2.1 (PMJ estimation) Given a set $\mathcal{P}$ of tuples $\left(\mathbf{p}_{l}, t_{l}\right) \in \Omega \times \mathbb{R}, l=1, \ldots, m$, where $t_{l}$ is the known activation time at $\mathbf{p}_{l}$, find a set $\mathcal{F}$ of estimated PMJs and associated activation times $\left(\hat{\mathbf{s}}_{i}, \hat{\tau}_{i}\right), i=1, \ldots, r$, that minimises the error function

$$
E=\frac{1}{m} \sum_{l=1}^{m}\left(t_{l}-\hat{t}_{l}\right)^{2}
$$

where $\hat{t}_{1}$ is the estimated activation time defined by (1), for $\mathbf{x}=\mathbf{p}_{/}$and the min function ranging in $\left(\hat{\mathbf{s}}_{i}, \hat{\tau}_{i}\right), i=1, \ldots, r$.

This problem admits a trivial solution consisting on taking $\mathcal{F}=\mathcal{P}$. If we apply a general unconstrained optimization method using (2) as cost function we shall find that many of the estimated points are located at positions belonging to $\mathcal{P}$. Moreover, the existence of this alternative solution shows that the problem is not convex, since it has several local minima. Thus we need a method to build $\mathcal{F}$ that avoids this solution.

Let us consider a PMJ with spatial coordinates $\mathbf{s} \in \Omega$ and activation time $\tau$. And let us consider a point $\mathbf{p}$ that is activated by the propagation of the signal from $\mathbf{s}$. The activation time $t$ of point $\mathbf{p}$ must meet the equation

$$
\|\mathbf{p}-\mathbf{s}\|=v(t-\tau)
$$

Equation (3) defines the positive half of a cone (see Figure 1, right, for a two dimensional representation of this idea) with its vertex in $(\mathbf{s}, \tau)$; thus, for any point activated by $\mathbf{s}$, the point $(\mathbf{p}, t) \in \mathbb{R}^{3}$ belongs to that cone. Given three points activated by the same PMJ, if a cone can be found in $\mathbb{R}^{3}$ containing their coordinates in the form $\left(\mathbf{p}_{/}, t_{l}\right)$, then PMJ coordinates (s, $\tau$ ) must be located at the cone's vertex. Moreover, since the activation of the point $\mathbf{p}$ is the result of a signal propagation, then its activation time $t$ will always be greater than the activation of the PMJ that caused it, $\tau \leq t$ (it would be equal only if $\mathbf{p}=\mathbf{s}$ ).

To construct a set of candidate PMJs, we are going to use these properties. As a first step in our method we build a Delaunay triangulation for the set of measured points, considering their spatial coordinates. Such a triangulation is the construction of an irregular mesh that takes the points as vertexes and in which all the faces are triangles [13]. Figure 2 shows the Delaunay triangulations for the smallest and largest sizes of $\mathcal{P}$, considered in our experiments.

Let's call

$$
\mathcal{T}=\left\{\left\{k_{1}, k_{2}, k_{3}\right\}: \text { The triangle formed by } \mathbf{p}_{k_{1}}, \mathbf{p}_{k_{2}}, \mathbf{p}_{k_{3}} \text { belongs to the Delaunay triangulation. }\right\}
$$



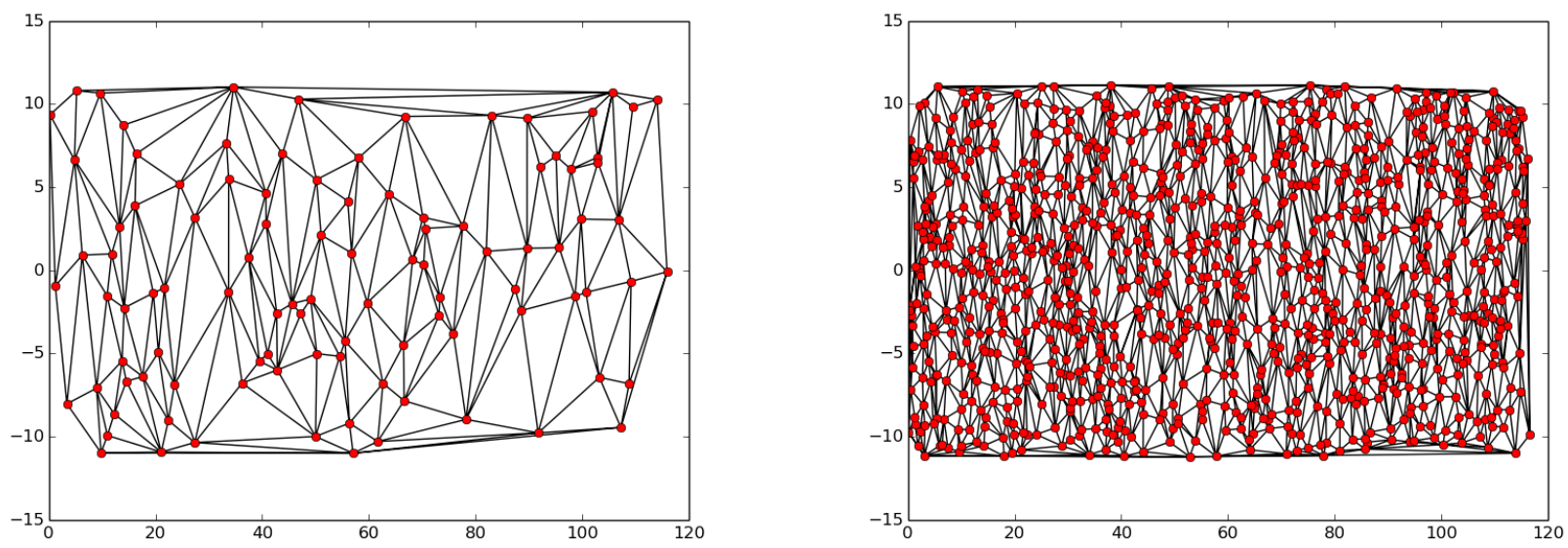

Figure 2. Two different measurement point sets, $\mathcal{P}$, and their corresponding Delaunay triangulations. (Left: $|\mathcal{P}|=100$, right: $|\mathcal{P}|=1000$ ).

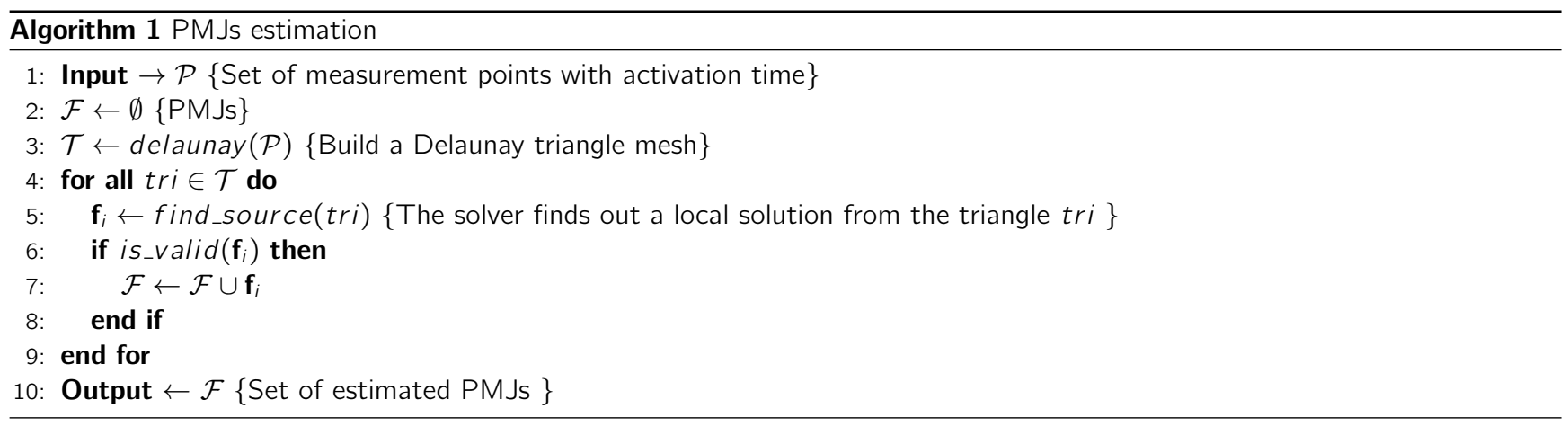

given a triangle $i=\left\{i_{1}, i_{2}, i_{3}\right\} \in \mathcal{T}$, formed by the measurement points, $\mathbf{p}_{i_{1}}, \mathbf{p}_{i_{2}}$ and $\mathbf{p}_{i_{3}}$, with activation times, $t_{i_{1}}, t_{i_{2}}$ and $t_{i_{3}}$, we look for a solution $\mathbf{f}_{i}=\left(\hat{\mathbf{s}}_{i}, \hat{\tau}_{i}\right) \in \mathbb{R}^{3}$ of the system of nonlinear equations

$$
\begin{aligned}
& \left\|\mathbf{p}_{i_{1}}-\hat{\mathbf{s}}_{i}\right\|-v\left(t_{i_{1}}-\hat{\tau}_{i}\right)=0, \\
& \left\|\mathbf{p}_{i_{2}}-\hat{\mathbf{s}}_{i}\right\|-v\left(t_{i_{2}}-\hat{\tau}_{i}\right)=0, \\
& \left\|\mathbf{p}_{i_{3}}-\hat{\mathbf{s}}_{i}\right\|-v\left(t_{i_{3}}-\hat{\tau}_{i}\right)=0 .
\end{aligned}
$$

Moreover, since a valid PMJ will have an activation time earlier than the LAT of the three measurements points that generate it, namely

$$
\tau_{i} \leq t_{i_{j}}, \quad j=1,2,3,
$$

the solution we find must meet this constraint.

\subsection{Algorithm description}

To extract a set $\mathcal{F}$ containing valid PMJs belonging to the hidden tree, we have designed Algorithm 1. For each triangle $i$ included in the Delaunay triangulation $\mathcal{T}$ we look for a valid tentative PMJ, $\mathbf{f}_{i}$. This step, corresponding to line 5 in the algorithm, consists of the solution of the system (4). By means of a change in the reference system, and a transformation to polar coordinates, this system can be solved analytically.

The previous step can lead to a false PMJ. This will happen when three points that form a triangle in the Delaunay triangulation were not activated by the same real PMJ. Under this situation, a candidate point $\mathbf{f}_{i}$ assumes that the three points were activated from a single source, while they were actually activated by two or three sources. To reduce the number of spurious PMJs, $\mathbf{f}_{i}$ 
needs to be validated. The validation function, called in line 6 of the algorithm, checks four conditions and only accepts the candidate PMJ if all of them are met.

The first condition checked is the verification that the candidate PMJ is consistent with the three measurement points that have generated it by verifying the conditions (5). The second condition checked is whether the estimated PMJ is inside the Delaunay triangulation $\mathcal{T}$, otherwise it is considered to be outside the domain of the problem and is disregarded. The third condition requires that the estimated PMJ is compatible with the backward eikonal problem associated to the triangle where the estimated PMJ is located in. This criterion has already been used by other authors [9] and states that a measurement point cannot activate later than the traveling wavefront produced by the closest PMJ. Thus, we request that the vertexes of triangle $k$ containing $\mathbf{f}_{i}=\left(\hat{\mathbf{s}}_{i}, \hat{\tau}_{i}\right)$ meet the condition

$$
t_{k_{j}} \leq \frac{\left\|\hat{\mathbf{s}}_{i}-\mathbf{p}_{k_{j}}\right\|}{v}+\hat{\tau}_{i}+\epsilon, \quad j=1, \ldots, 3
$$

where $\epsilon$ is a tolerance parameter that accounts for possible numerical errors.

The last condition requires to find a new measurement point satisfying equation (4). Therefore, the total number of points required to validate a PMJ candidate is 4 (3 points from the Delaunay triangle and an extra one).

\subsection{Discussion of the method}

The proposed method takes as an input a set of measurement points in $\Omega$ with their associated activation times. The detection of a given $\mathrm{PMJ}, \mathbf{s}_{i} \in \mathcal{S}$, depends on the fact that at least one triangle of $\mathcal{T}$ is contained in the region

$$
V\left(\mathbf{s}_{i}\right)=\left\{\mathbf{x} \in \Omega:\left\|\mathbf{s}_{i}-\mathbf{x}\right\|+v \tau_{i} \leq\left\|\mathbf{s}_{k}-\mathbf{x}\right\|+v \tau_{k}, \forall\left(\mathbf{s}_{k}, \tau_{k}\right) \in \mathcal{S}\right\}
$$

which is the additively weighted Voronoi region associated to $\mathbf{s}_{i}$ [13]. For the sake of brevity we shall denote it by $V_{i}$. In the absence of further assumptions about the data acquisition procedure, we will consider that the spatial locations of measurement points are a random sample of a uniform distribution on $\Omega$. As a consequence, the probability that a point lies on a given region is proportional to its area. The question that arises is whether the probability of not detecting a given PMJ can be made arbitrarily small by increasing the number of measurement points.

Let $\mathbf{s}_{i}$ be a PMJ with activation time $\tau_{i}$. The area of $V_{i}$ depends on the relative position and activation time of $\mathbf{s}_{i}$ respect to the other PMJs. If there exists some $\mathbf{s}_{j}$ with $\tau_{j}<\tau_{i}$ such that $\left\|\mathbf{s}_{i}-\mathbf{s}_{j}\right\|<\tau_{i}-\tau_{j}$, then the signal from $\mathbf{s}_{j}$ will arrive in $\mathbf{s}_{i}$ before it activates. As a consequence, $V\left(\mathbf{s}_{i}\right)=\emptyset$ and no tissue will be activated from $\mathbf{s}_{i}$, making it undetectable. A similar condition appears when there exists some PMJ $\mathbf{s}_{j}$, with $\tau_{j}<\tau_{i}$, such that $\left\|\mathbf{s}_{i}-\mathbf{s}_{j}\right\|=\tau_{i}-\tau_{j}$. In this case, the region activated by $\mathbf{s}_{i}$ is a line segment that starts at $\mathbf{s}_{i}$ and it spans in the opposite direction from $\mathbf{s}_{j}$. Since the Voronoi region of $\mathbf{s}_{i}$ has, in this case, null measure, it is undetectable by our method. Anyway, this PMJ is indistinguishable from $\mathbf{s}_{j}$ and, it has no effect on tissue activation (if we remove it, the activation time at any location does not change due to $\mathbf{s}_{j}$ ).

These layouts are not relevant to our goal, since the activation map is not affected at all by PMJs that fulfill any of these conditions. They are not effective, in the sense that they do not change the activation time at any location of the heart tissue. In what remains, we will call effective PMJ to any signal source point that is not in any of the two cases described above. That is, a PMJ with location $\mathbf{s}_{i}$ and activation time $\tau_{i}$ is said to be effective if there is no other PMJ, $\mathbf{s}_{j}$ with $\tau_{j}<\tau_{i}$, such that $\left\|\mathbf{s}_{i}-\mathbf{s}_{j}\right\| \leq \tau_{i}-\tau_{j}$. In this work we focus only on the estimation of effective PMJs.

Let $\mathbf{s}_{i}$ be an effective PMJ. For $\mathbf{s}_{i}$ not to be detected, it is necessary that there is no triangle of the Voronoi triangulation $\mathcal{V}$ with its three vertexes in $V_{i}$. This would happen either if there is less than three measurement points in $v_{i}$ or if, having three or more points in $V_{i}$, they would not form a triangle in $\mathcal{V}$.

Let us first consider the probability that no three points fall in $V_{i}$. Let $A_{i}$ be the area of $V_{i}$ and $A$ the area of the whole problem domain $\Omega$, the probability that a single point is in $V_{i}$ will be $p_{i}=A_{i} / A$. If a set $\mathcal{P} \subset \Omega$ with $m \geq 3$ random points is generated, we can consider the random variable $X_{i}=\left|\left\{(\mathbf{p}, t) \in \mathcal{P}: \mathbf{p} \in V_{i}\right\}\right|$, where $|\cdot|$ denotes cardinal, which follows a Binomial distribution $X_{i} \sim B\left(m, p_{i}\right)$. The probability that less than 3 points are in $V_{i}$ is, then,

$$
P\left(X_{i} \leq 2\right)=\left(1-p_{i}\right)^{m}+p_{i}\left(1-p_{i}\right)^{m-1}+p_{i}^{2}\left(1-p_{i}\right)^{m-2}
$$




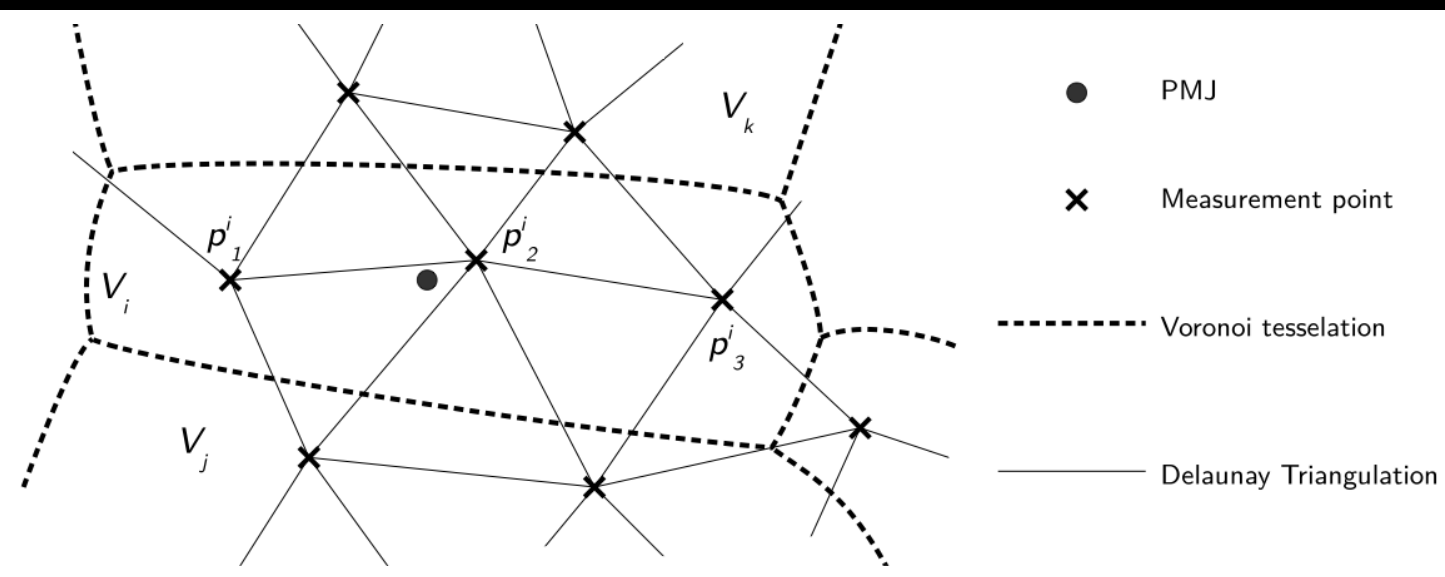

Figure 3. A situation with a Voronoi region, $V_{i}$, containing three measurement points, $\mathrm{p}_{1}^{i}, \mathrm{p}_{2}^{i}$ and $\mathrm{p}_{3}^{i}$, which do not form a triangle of the Delaunay triangulation.

which tends to 0 as $m \rightarrow \infty$.

Next, we focus in the case when we have three or more points inside $V_{i}$ and they do not form a triangle in $\mathcal{V}$. An example of a set of measurement points that leads to this situation with three points is shown in Figure 3 . Let $\mathbf{p}_{1}^{i}, \mathbf{p}_{2}^{i}, \ldots, \mathbf{p}_{k}^{i}$ be a set of $k \geq 3$ measurement points which are inside $V_{i}$ but do not generate any triangle in $\mathcal{V}$, and let $H$ be the area of the convex hull formed by them. If any new measurement point is added which is contained in $H$, then the new set of points in $V_{i}$ will form two or more new triangles in $\mathcal{V}$ [13]. Since $H$ is nonzero with probability 1 , following the previous reasoning, we can see that the probability of this not happening tends to 0 as $m$ grows.

From previous analysis, it is derived that the probability of not detecting a given PMJ tends to zero if we are capable of increasing the number of measurement points. An additional property that arises from this discussion is that, the bigger the area a given PMJ activates, the higher the probability of being detected by the algorithm. Since in many clinical situations the interest is in knowing the origin of the activation of a given tissue region, this indicates that our method will detect the most relevant PMJs first with higher probability.

Another possible source of error in the algorithm is the estimation of a PMJ that actually is not in $\mathcal{S}$. This can happen if we estimate a PMJ from a triangle with vertexes belonging to different Voronoi regions. However, as discussed in Section 2.1, before a tentative PMJ is accepted several validations tests are performed, which reduce the number of spurious estimations.

\section{Performance Evaluation}

To evaluate our approach, we have generated several scenarios consisting of a simulated Purkinje tree structure along with a set of measurement points uniformly distributed. In this section we describe the experimental methodology used and discuss the results. We provide several performance measurements for our method, together with a comparison with the methodology proposed by Cardenes et al. [7].

\subsection{Methodology}

The test trees representing the cardiac conduction system are built procedurally. We use a recursive algorithm that, at every level, creates two new branches at the end of each branch that was built in the previous level. Every new branch is perpendicular to the parent branch. The leaf nodes of the tree are the PMJs, which represent sources of electrical signal in the myocardium. The density of PMJs is indirectly controlled by the depth of the branch recursion. Branch lengths are generated following a normal distribution with parameters obtained from [4]. Figure 4 shows two examples of trees used in our experiments.

We generate two types of scenarios: i) trees with a single main branch, and ii) trees with three main branches connected as depicted in Figure 4. For each scenario type, we consider 3 different recursion depths for generating tree subbranches: depth 2, depth 4 and depth 6 . We will label every configuration with letter B followed by the number of branches plus letter $D$ followed 


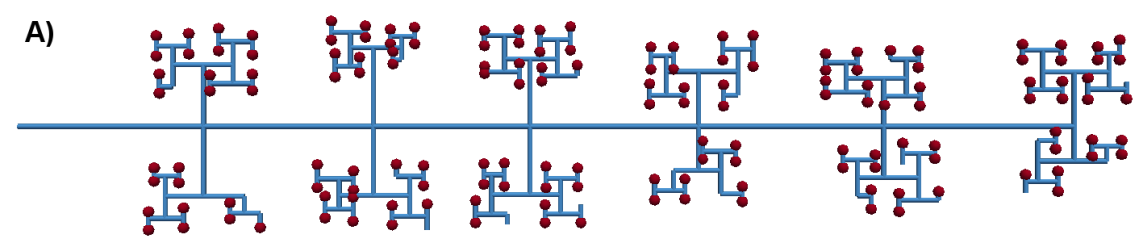

B)

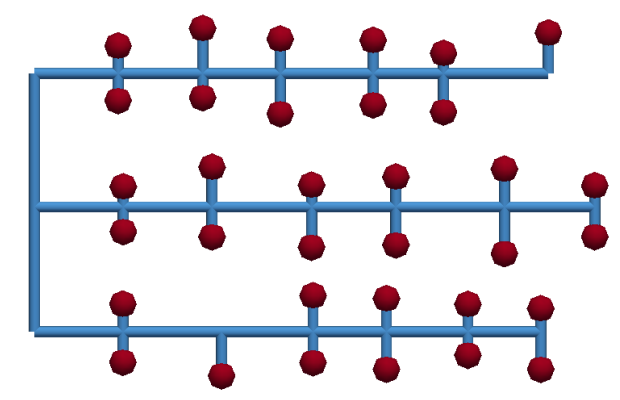

Figure 4. Example of generated trees. A) 1 main branch with depth 6 (B1D6), B) 3 main branches with depth 2 (B3D2)

by the depth used to generate it. E.g., a tree with 3 branches and depth 4 will be named B3D4. Figure 4 (A) shows a tree with one branch and recursion depth of 6 (B1D6). Figure 4 (B) shows a tree with three branches and a recursion depth of 2 (B3D2).

Given a simulated Purkinje tree, we firstly place a set of measurement points in the domain, uniformly distributed, with a number of points varying from 100 to 1000 in steps of 100 . Secondly, the LAT at each measurement point is computed, by propagating the electrical signal along the tree. This propagation is done first from the tree root up to the PMJs and then from the PMJs to each sensor through the shortest path, using Equation (1). The signal propagates within the tree around three times faster than on the myocardial tissue domain. The experimental process can be summarized as follows: i) Generate an artificial tree with a set of PMJs as source points, $\mathcal{S}$; ii) Generate a set $\mathcal{P}$ of measurement points uniformly distributed; iii) Propagate the signal from $\mathcal{S}$ to $\mathcal{P}$ and set the corresponding activation times for every point in $\mathcal{P}$; iv) Run the algorithm proposed in Section 2.1 and obtain an estimation $\mathcal{F}$; v) Compare the solution $\mathcal{S}$ with the estimation $\mathcal{F}$ and evaluate its quality.

In Figure 5, the results of the algorithm can be observed for two dense scenarios (B3D4 and B3D6), with two different measurement points densities. In the figure, measurement points are represented by crosses. The blue dots represent the PMJs that have been found, while the red dots represent PMJs that have not been found in the solution by our algorithm. The set of all measurement points which are activated by a common PMJs is represented by a polygon enclosing them.

To evaluate these results we consider two approaches. First, we compare the generated PMJs $\mathcal{S}$ (considered as the true unknown cardiac conduction system) and the estimation $\mathcal{F}$ provided by our algorithm. This will give us information about the ability of the algorithm to find the actual PMJs in the simulated scenarios. This quality measurement is only available because we have the actual (generated) PMJs of the CCS. When the method is applied to clinical data, real PMJs will not be available for comparison. Then, as a second error measure we compare the activation map generated by $\mathcal{F}$ to the activation times measured in the locations of $\mathcal{P}$. Following previous works $[8,9]$, we use the maximum absolute error and the mean absolute error.

To be able to understand the quality of the results, it is important to define what is an acceptable error in our problem. The physiological signal propagation process that motivates this work starts when the electrical signal enters the CCS and ends when the whole cardiac tissue has been activated. In our synthetic cardiac tissue, this process cannot last more than $134 \mathrm{~ms}$, which corresponds to the diameter of the domain divided by the propagation velocity of the signal in the tissue. It is also important to know that in the literature an error below $5 \mathrm{~ms}$ is considered a highly accurate LAT $[14,15]$. 


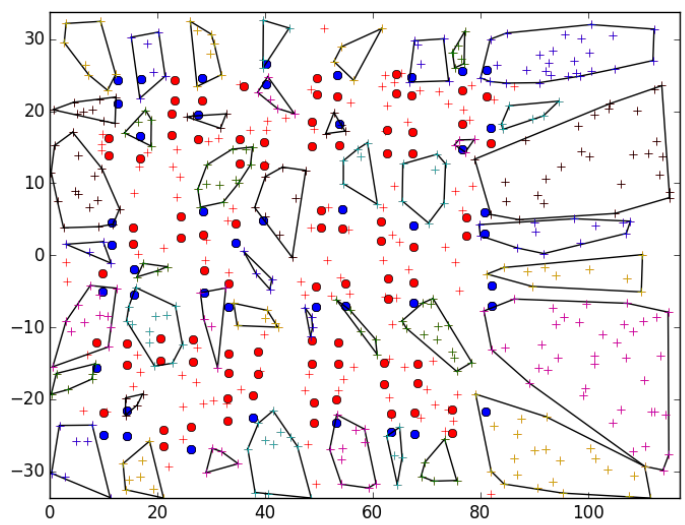

(a)

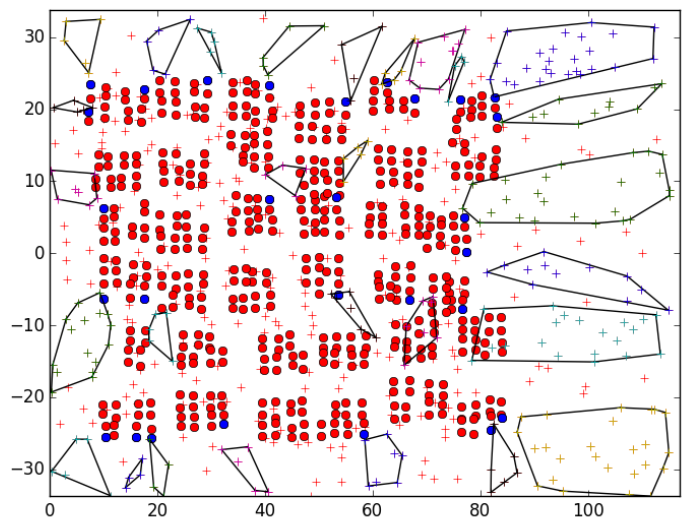

(c)

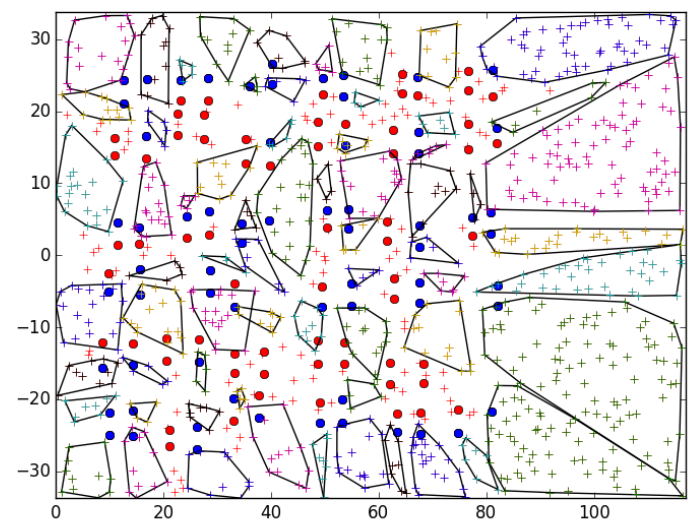

(b)

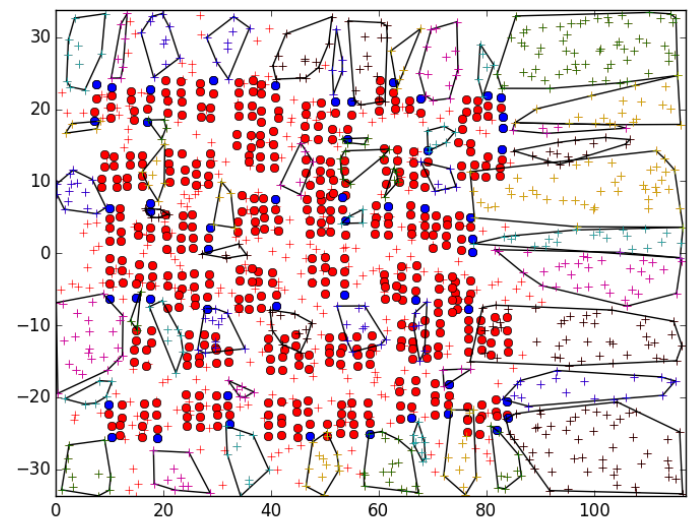

(d)

Figure 5. Solutions provided for scenario B3D4 (top) and B3D6 (bottom) with (a,c) 500 measurement points and (b,d) 1000 measurement points

\subsection{Results and discussion}

The results of the simulation study, including all the scenarios, are summarized in Figure 6 . The number of sources, i.e. PMJs, estimated for the scenario with a single main branch is displayed in Figure 6 (a,c,e), while Figure 6 (b,d,f) shows the PMJs estimated using three main branches. Every row represents a different recursion depth, for depths 2, 4 and 6 . Each plot shows the number of PMJs in the scenario (F_REAL), the number of PMJs that can be truly estimated (F_DET) or effective sources, the total number of PMJs estimated by the algorithm (F_EST) and finally, the number of PMJs correctly estimated (F_OK).

In Figure 6, there is a small difference between the values of the estimated (F_EST) and correctly estimated (F_OK) number of PMJs which indicates that some false positives are obtained (estimated PMJs in $\mathcal{F}$ that were not in $\mathcal{S}$ ). In configurations where measurement points are not activated by the same PMJ, but produce a feasible candidate we obtained false positives. Although several validation tests are performed during the algorithm, as discussed in Section 2, the results show that the validation function does not detect all of them. In scenarios B1D2, B1D4 and B3D2 the algorithm obtains nearly all PMJs when it has enough measurement points. However, when the density of PMJs increases the problem becomes more complex, since these points tend to be clustered, as Figure 5 shows.

Figure 7 (a) shows the number of PMJs estimated versus the number of real PMJs for a fixed number of 1000 measurement points. Notice that each point in the plot corresponds to one scenario (B1D2, B3D2, B1D4, B3D4, B1D6 and B3D6) with it corresponding number of PMJs. In the first four scenarios, the number of estimated PMJs increases with the total number of PMJs. However, this does not occur in the last two scenarios (B1D6, B3D6) where the number of found PMJs decreases. This 


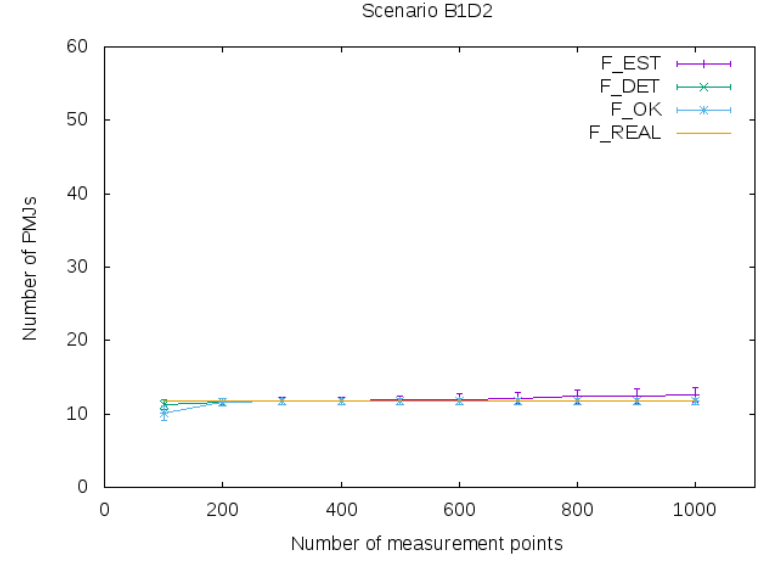

(a)

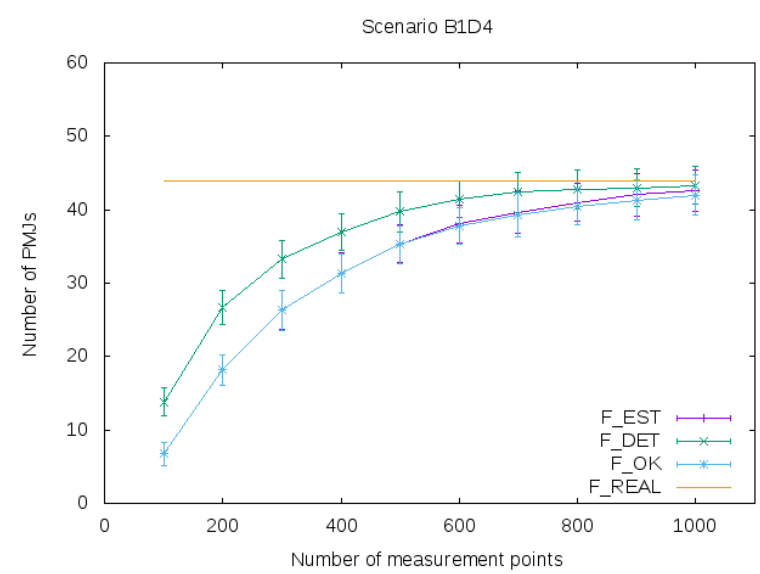

(c)

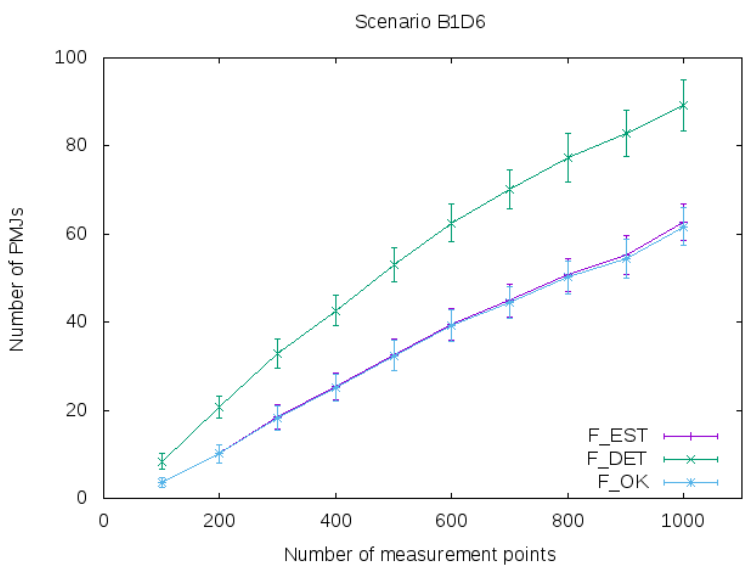

(e)

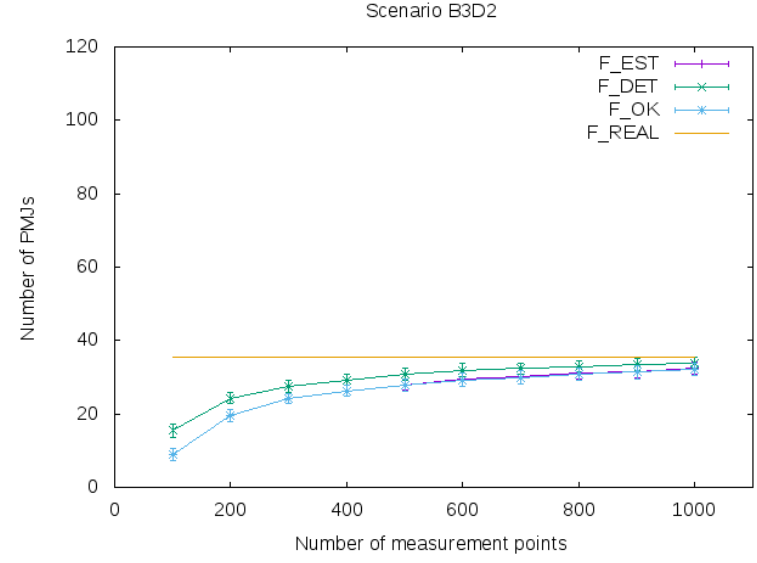

(b)

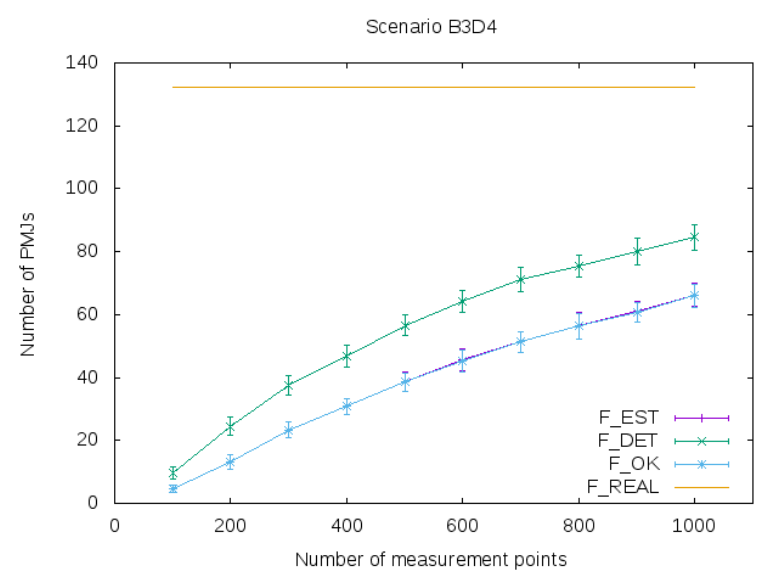

(d)

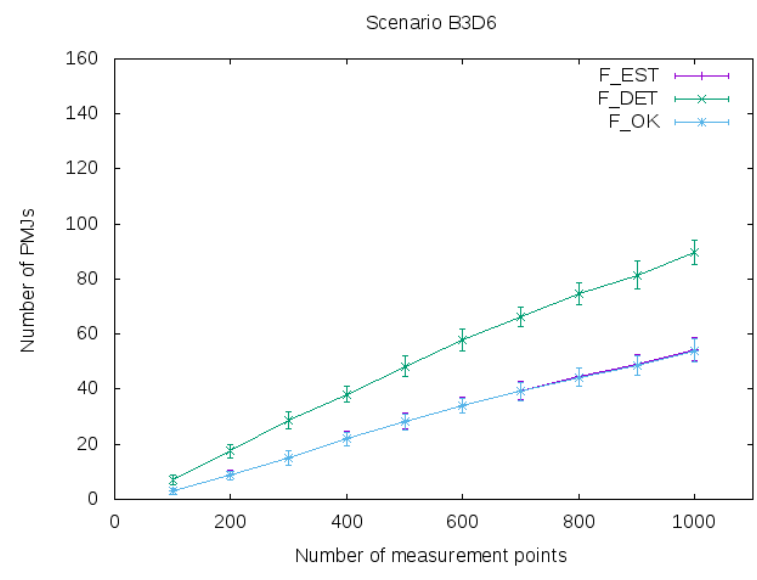

(f)

Figure 6. Results for $(\mathrm{a}, \mathrm{c}, \mathrm{e})$ a single main branch, and (b,d,f) for three main branches. Every row corresponds to a different recursion depth. The used recursion depths are (from top to bottom) 2, 4 and 6. The results are averaged for the different instances of each scenario, and plotted with its standard deviation. Figures are not at the same scale, since the number of PMJs varies for the different scenarios. See the text for details on the figure contents.

behaviour is also amplified because we have set a fixed number of measurement points. As a consequence, the ratio between measurement points and PMJs decreases, making it more difficult to build a complete estimate.

Despite these apparent limitations, Figure 7 (b) shows how the mean of the absolute error associated to each scenario decreases rapidly with the measurement points and from 500 measurement points and on, it stays below $2 \mathrm{~ms}$ for all scenarios. 


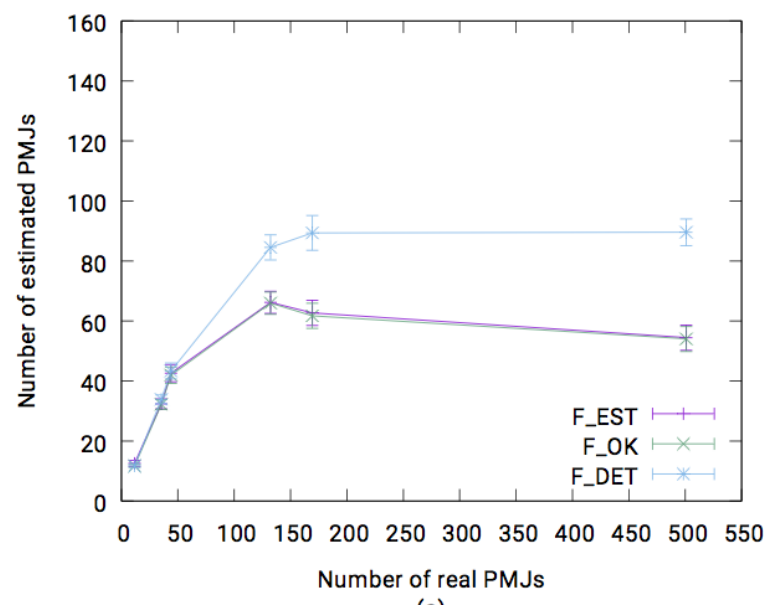

(a)

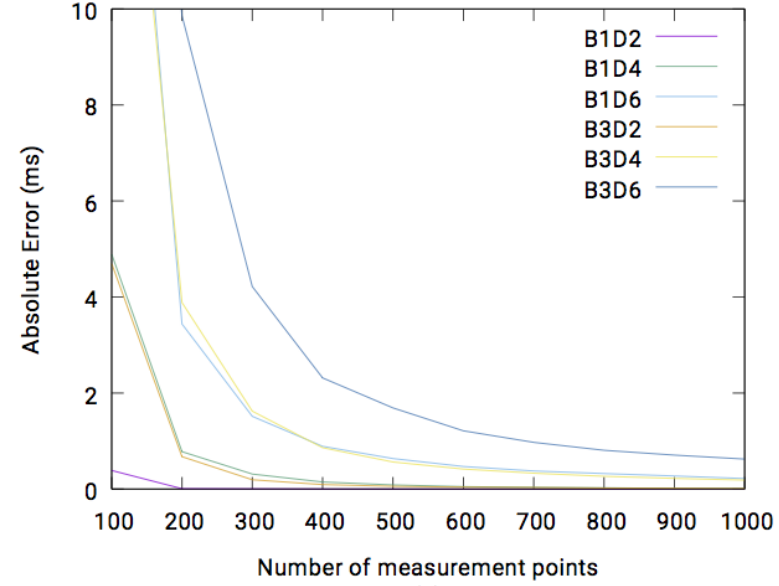

(b)

Figure 7. (a) Number of PMJs detected with respect to the total number of PMJs with 1000 measurement points. (b) The mean of the absolute error (in milliseconds) for the different scenarios considered.

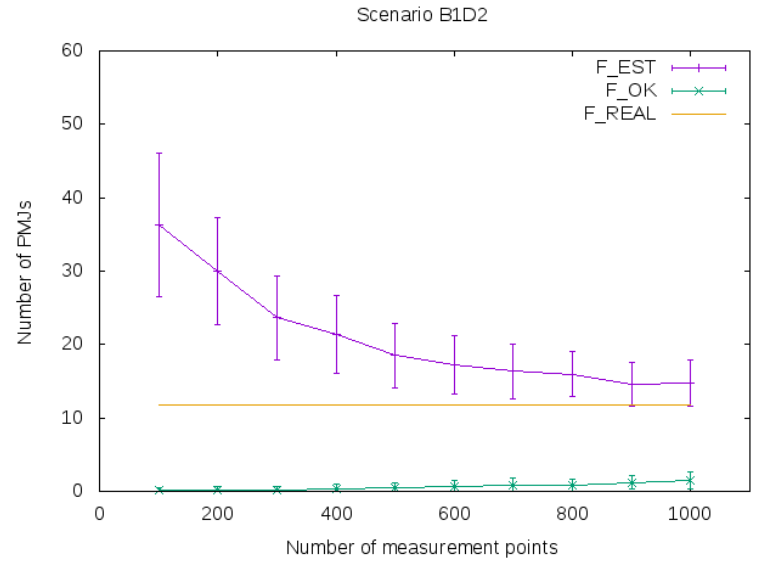

(a)

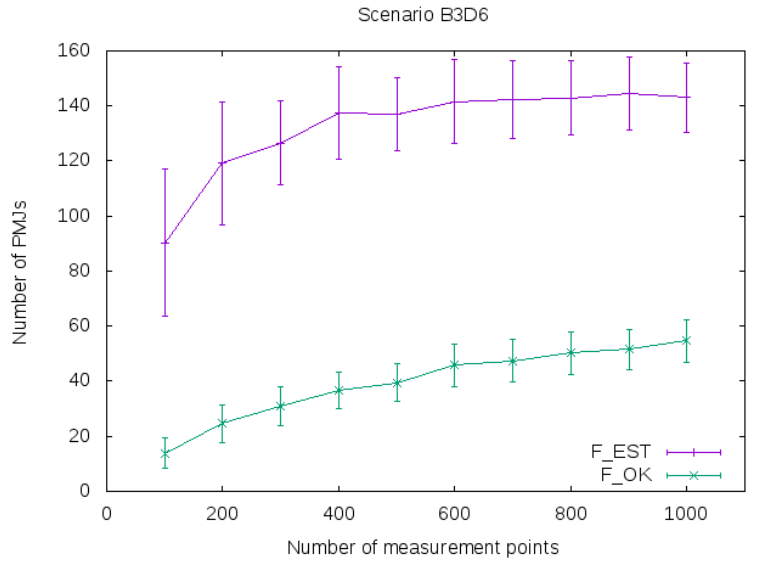

(b)

Figure 8. Estimation results for scenarios B1D2 (a) and B3D6 (b) using the gradient method.

This behavior of the error reveals that the correctly estimated PMJs are the most significant ones. As can be seen in Figure 5, the PMJs which are properly detected are close to the border of the region occupied by the tree, while the inner PMJs are those missed by the algorithm. The signal emitted by these inner points is quickly masked by the points at the border of the tree and they are mainly non-effective PMJs. From these results, we conclude that our algorithm is capable of finding most of the effective nodes of the Purkinje system.

To compare our method with previous approaches, we have implemented the method described in [7] based on the gradient of the activation time considered as a differentiable function of the location. In Figure 8 we present the two extreme scenarios, B1D2 and B3D6, solved with this method, in Figure 9 (a) we show the total number of estimated PMJs and the number of correctly estimated PMJs and in Figure 9 (b) we can see the mean of the absolute error for the different scenarios. It is remarkable that in the simplest scenario, B1D2, the method performs poorly, many PMJs are detected but only few are correct, producing a high absolute error. Nevertheless, with the most complex scenario, B3D6, the number of correctly estimated PMJs is similar to that obtained with our method.

In summary, our method has shown a higher accuracy at estimating PMJs and it performs well with low and high densities of PMJs. On the other hand, the gradient method has obtained lower errors for scenarios with high densities of PMJs and low density of measurement points. 


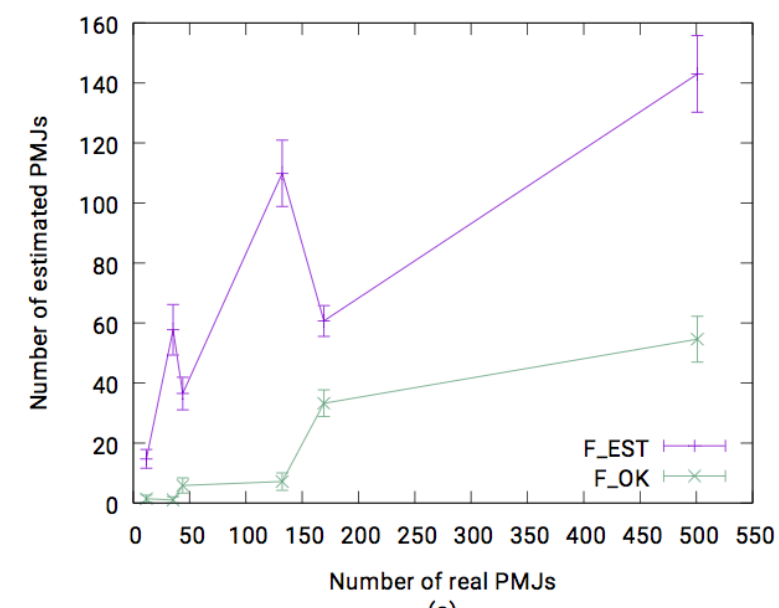

(a)

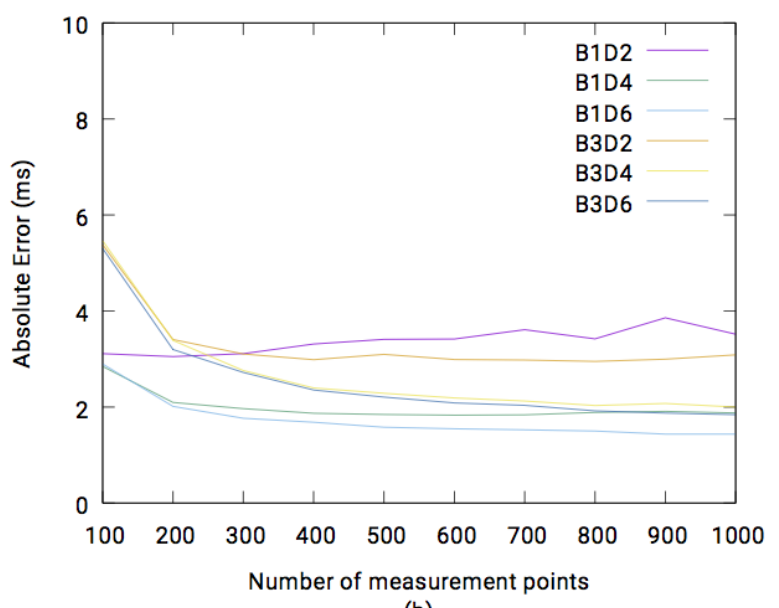

(b)

Figure 9. Results for the gradient method: (a) Number of PMJs detected with respect to the total number of PMJs with 1000 measurement points. (b) The mean of the absolute error (in milliseconds) for the different scenarios considered.

\section{Conclusions}

We have presented a novel methodology to estimate, from electro-anatomical maps, the location and activation time of the sources of electrical activation, known as PMJs, in a simplified model of cardiac tissue. Therefore, using the location and the corresponding activation times of these estimated PMJs, we can obtain the activation map required (simulating a diffusion from these PMJs to the tissue considered).

The following conclusions can be dragged from the numerical results shown in the previous section. The proposed method can detect any PMJ if enough measurement points are available, that is, when the scenario has an appropriate ratio between the number of PMJs and samples acquired. We have observed that in most scenarios, in order to detect all PMJs, we will need to sample, at least, with a density three times higher than the highest density of PMJs. When clustered PMJs appear, many of them are virtually impossible to be detected with the current setup. However, in those scenarios the method still shows a good performance, since many clustered PMJs are non-effective or have a very local impact in the overall activation map. This is confirmed by the mean absolute errors obtained when using estimated PMJs, which are low in all the scenarios (including clustered) for EAMs with more than 600 samples acquired. With low density EAMs ( $<250$ samples) the method might perform poorly in dense PMJ scenarios. Compared to previous methods [7], our method outperforms in all scenarios tested when the number of measurement points is greater than 250 .

The method presented does not address the recovery of the Purkinje tree structure, and therefore it cannot be used in pathological scenarios in which PMJs are activated retrogradely, i.e. from tissue to the Purkinje tree. However, results of our algorithm can be used as an input to Purkinje tree estimation methods.

The next step will be to take into account the anisotropic properties of the underlying tissue where the PMJs are placed, and the Purkinje tree structure. Other main future research directions are: to build a robust version of the algorithm, capable of estimating the PMJs from noisy LAT data, and to extend the domain to arbitrary geometries.

\section{Acknowledgements}

This work was supported by Ministerio de Economa y Competitividad and the European Commission (European Regional Development Funds - ERDF - FEDER) with grant numbers TIN2014-59932-JIN and TIN2015-66972-C5-5-R. 


\section{References}

1. Lopez-Perez A, Sebastian R, Ferrero JM. Three-dimensional cardiac computational modelling: methods, features and applications. Biomedical engineering online 2015; 14:35.

2. Boyle PM, Mass S, Nanthakumar K, Vigmond EJ. Transmural ik(atp) heterogeneity as a determinant of activation rate gradient during early ventricular fibrillation: mechanistic insights from rabbit ventricular models. Heart Rhythm Nov 2013; 10(11):1710-1717.

3. Behradfar E, Nygren A, Vigmond EJ. The role of purkinje-myocardial coupling during ventricular arrhythmia: A modeling study. PLoS ONE 02 2014; 9(2):e88000.

4. Sebastian R, Zimmerman V, Romero D, Sanchez-Quintana D, Frangi AF. Characterization and modeling of the peripheral cardiac conduction system. IEEE Trans Med Imaging Jan 2013; 32(1):45-55.

5. Liu BR, Cherry EM. Image-Based Structural Modeling of the Cardiac Purkinje Network. BioMed Research International 2015; 2015:15, doi: $10.1155 / 2015 / 621034$.

6. Stephenson RS, Boyett MR, Hart G, Nikolaidou T, Cai X, Corno AF, Alphonso N, Jeffery N, Jarvis JC. Contrast enhanced microcomputed tomography resolves the 3-dimensional morphology of the cardiac conduction system in mammalian hearts. PLOS ONE 04 2012; 7(4):e35 299, doi:10.1371/journal.pone.0035299.

7. Cárdenes R, Sebastian R, Soto-Iglesias D, Berruezo A, Camara O. Estimation of Purkinje trees from electro-anatomical mapping of the left ventricle using minimal cost geodesics. Medical image analysis Aug 2015; 24(1):52-62.

8. Vergara C, Palamara S, Catanzariti D, Nobile F, Faggiano E, Pangrazzi C, Centonze M, Maines M, Quarteroni A, Vergara G. Patientspecific generation of the purkinje network driven by clinical measurements of a normal propagation. Med Biol Eng Comput. 2014; 52(10):813-26.

9. Palamara S, Vergara C, Faggiano E, Nobile F. An effective algorithm for the generation of patient-specific purkinje networks in computational electrocardiology. Journal of Computational Physics 2015; 283:495 - 517, doi:http://dx.doi.org/10.1016/j.jcp.2014. 11.043.

10. Romero D, Sebastian R, Bijnens BH, Zimmerman V, Boyle PM, Vigmond EJ, Frangi AF. Effects of the purkinje system and cardiac geometry on biventricular pacing: a model study. Ann Biomed Eng Apr 2010; 38(4):1388-1398, doi:10.1007/s10439-010-9926-4.

11. Hyde ER, Behar JM, Claridge S, Jackson T, Lee AW, Remme EW, Sohal M, Plank G, Razavi R, Rinaldi CA, et al.. Beneficial effect on cardiac resynchronization from left ventricular endocardial pacing is mediated by early access to high conduction velocity tissue: Electrophysiological simulation study. Circulation: Arrhythmia and Electrophysiology 2015; 8(5):1164-1172, doi: 10.1161/CIRCEP.115.002677.

12. Atkinson A, Inada S, Li J, Tellez JO, Yanni J, Sleiman R, Allah EA, Anderson RH, Zhang H, Boyett MR, et al.. Anatomical and molecular mapping of the left and right ventricular His-Purkinje conduction networks. J Mol Cell Cardiol Jun 2011; 51(5):689-701.

13. Deza E, Deza MM. Dictionary of Distances. Elsevier, 2006.

14. Haddad ME, Houben R, Stroobandt R, Heuverswyn FV, Tavernier R, Duytschaever M. Algorithmic detection of the beginning and end of bipolar electrograms: Implications for novel methods to assess local activation time during atrial tachycardia. Biomedical Signal Processing and Control 2013; 8(6):981 - 991, doi:http://dx.doi.org/10.1016/j.bspc.2012.11.005. URL http://www. sciencedirect. com/science/article/pii/S174680941200119X.

15. Alcaine A, Soto-Iglesias D, Calvo M, Guiu E, Andreu D, Fernndez-Armenta J, Berruezo A, Laguna P, Camara O, Martnez JP. A waveletbased electrogram onset delineator for automatic ventricular activation mapping. IEEE Transactions on Biomedical Engineering 2014; 61(12):2830-2839, doi:10.1109/TBME.2014.2330847. 